\title{
The Dstpk61 locus of Drosophila produces multiple transcripts and protein isoforms, suggesting it is involved in multiple signalling pathways
}

\author{
D Clyde and M Bownes
}

The University of Edinburgh, Institute of Cell and Molecular Biology, Darwin Building, King's Buildings, Mayfield Road, Edinburgh EH9 3JR, UK

(Requests for offprints should be addressed to M Bownes; Email: Mary.Bownes@ed.ac.uk)

\begin{abstract}
The Drosophila gene Dstpk61 encodes a serine threonine protein kinase homologous to human phosphoinositidedependent protein kinase (PDK1), and also has homologues in S. cerevisiae, S. pombe, C. elegans, A. thaliana, mouse, and sheep. Where its function has been investigated, this kinase is thought to be involved in regulating cell growth and survival in response to extracellular signals such as insulin and growth factors. In Drosophila it produces multiple transcripts, some of which appear to be sex-specific.

In addition to the five Dstpk61 cDNAs we have described previously we report the existence of a further 18 expressed sequence tag (EST) cDNAs, three of which we have fully sequenced. We conclude that Dstpk61 is a complex locus that utilises a combination of alternative
\end{abstract}

promoters, alternative splice sites and alternative polyadenylation sites to produce a vast array of different transcripts. These cDNAs encode at least four different DSTPK61 protein isoforms with variant $\mathrm{N}$-termini. In this paper, we discuss the possible functions of the distinct Dstpk61 transcripts and how they might be differentially regulated. We also discuss the roles that DSTPK61 protein isoforms might play in relation to the protein domains they contain and their potential targets in the cell. Finally, we report the putative structure of the human PDK1 gene based on computer comparisons of available mRNA and genomic sequences. The value of using sequence data from other species for experimental design in mammalian systems is discussed.

Journal of Endocrinology (2000) 167, 391-401

\section{Introduction}

Signalling cascades are a crucial means of cell-cell communication in all types of organisms. During development, cells need to communicate with each other in order to form a structured organism, with each cell knowing its identity and role. Many of these pathways form evolutionarily conserved cassettes, where homologous proteins perform equivalent roles in different organisms. For example, the EGFR signalling pathway is conserved in Drosophila and mammals and regulates cell proliferation, differentiation and survival in both systems (Sibilia \& Wagner 1995, Van Buskirk \& Schupbach 1999).

Signalling cascades are also essential for cells to be able to respond to their environment. Multiple signalling pathways exist, each of which results in different cellular responses. However, some signalling proteins appear to be involved in more than one cascade. Such proteins act as branch points in the signalling hierarchy, so that a single agonist can activate a number of cascades and induce multiple cellular responses or different responses under different conditions. One such protein is human phosphoinositide-dependent kinase (PDK1), which has been shown to phosphorylate protein kinase A (PKA) (Cheng et al. 1998), PKB (Alessi et al. 1997b), PKC (Le Good et al. 1998), serum- and glucocorticoid-inducible kinase (SGK) (Park et al. 1999), and $\mathrm{p} 70^{\mathrm{S} 6 \mathrm{~K}}$ (Alessi et al. 1998, Pullen et al. 1998).

PDK1 has been most extensively studied for its ability to phosphorylate $\mathrm{PKB}$ in response to insulin (Alessi et al. 1997b). This phosphorylation only occurs in the presence of phosphatidyl inositol-4,5-bisphosphate and -trisphosphate $\left(\mathrm{PI}(3,4) \mathrm{P}_{2}\right.$ and $\mathrm{PI}(3,4,5) \mathrm{P}_{3}$ respectively), produced by PI $3-\mathrm{K}$. These membrane lipids interact with the $\mathrm{PH}$ domain of PDK1, presumably bringing it into proximity with membrane-bound $\mathrm{PKB}$ and allowing phosphorylation to occur (Currie et al. 1999). PKB phosphorylation evokes a number of cellular responses, including: increased glucose uptake and glycolysis; increased glycogen synthesis; increased protein synthesis and a concomitant cell cycle progression from G1 to S phase; and increased cell survival, via the repression of the pro-apoptotic proteins BAD and FKHRL1 (del Peso et al. 1997, Scheid \& Duronio 1998, Brunet et al. 
1999, Craddock et al. 1999). Interestingly, the Drosophila homologue of PKB, Dakt1, has also been implicated in promoting cell survival (Staveley et al. 1998).

Drosophila homologues have also been identified in the pathway upstream of PKB. CHICO is the Drosophila homologue of IRS1-4 and, when mutated, the resulting flies are viable but about one-third the size of wild-type adults (Bohni et al. 1999). Similarly, the Drosophila homologue of the p110 subunit of PI 3-K, Dp110, results in differences in cell size and number when mis-expressed in imaginal discs (Leevers et al. 1996). Thus, the insulin response pathway in Drosophila also appears to be involved in regulating cell growth and proliferation.

PDK1 has also been shown to phosphorylate PKA, also know as cAMP-dependent protein kinase. Signalling via mammalian PKA is complex - multiple isoforms are present, which respond differently to different extracellular stimuli (Skalhegg \& Tasken 1997). Thus, PKA elicits multiple biological effects, not all of which are dependent on cAMP - some responses are thought to act through NFKB:IкB (Zhong et al. 1997). In mammals, PKA signalling has effects on metabolism, gene regulation, cell growth and division, differentiation, sperm motility, and ion channel conductivity (Skalhegg \& Tasken 1997). Three genes encoding PKA catalytic subunits have been found in Drosophila: PKAc1, PKAc2 and PKAc3, also known as DCO,DC1, and DC2. As in mammals, these genes produce multiple transcripts (Kalderon 1997). Again, this reflects the multiple roles that PKA signalling plays in Drosophila, where it is involved in: segmentation, cell growth, oocyte polarity, learning and memory, and regulation of quantal size at the neuromuscular junction. Interestingly, PKA is thought to exert its effect on segmentation via hedgehog in a cAMP-independent manner (Ohlmeyer \& Kalderon 1997).

Many PKC isoforms are also phosphorylated by PDK1 (Le Good et al. 1998, Dong et al. 1999). These different isoforms vary considerably in their time, position and level of expression, reflecting the diverse cellular functions of PKC in cell transformation, growth, differentiation, membrane ruffling, vesicle trafficking, and gene expression (Liu \& Heckman 1998). Three PKC encoding genes have been identified in Drosophila: Pkc53E, Pkc98E, and inactivation no afterpotential $C$ (inaC). Pkc98E and Pkc53E are expressed only in the head, and InaC is detected only in the eye. These restricted expression patterns reflect the functions of PKC in Drosophila, where it is thought to be involved mainly in neural activation and learning and memory. However, it is possible that ubiquitously expressed PKC homologues remain to be identified in Drosophila. The complete Drosophila genome sequence is now available and reveals the presence of a few previously unidentified $P k c-$ like genes, although the expression patterns of transcripts from these loci remain to be investigated.

Mammalian $\mathrm{p} 70^{\mathrm{S} 6 \mathrm{~K}}$ is activated in response to insulin and mitogen stimulation, and results in the increased translation of $5^{\prime}$ TOP mRNAs. This set of mRNAs encodes mostly proteins of the translational machinery, such as ribosomal proteins. Their upregulation is essential for cell cycle progression from G1 to S phase, and is thus associated with the regulation of cell proliferation and/or differentiation (for review see Peterson \& Schreiber 1998). Full activation of $\mathrm{p} 70^{\mathrm{S} 6 \mathrm{~K}}$ requires phosphorylation by PDK1 (Alessi et al. 1998, Pullen et al. 1998). A Drosophila homologue of $p 70^{S 6 K}$ exists and is known as $D p 70^{S 6 K}$ or S6K (Stewart et al. 1996, Watson et al. 1996). Although no functional studies have been reported, it is interesting to note that $D p 70^{S 6 K}$ encodes two protein isoforms: 3.7 and $5.0 \mathrm{~kb}$ transcripts encode an identical 637 amino acid protein, whereas a $2.8 \mathrm{~kb}$ transcript encodes a smaller protein that lacks the $51 \mathrm{~N}$-terminal residues of the larger isoform (Watson et al. 1996, Stewart et al. 1996). The mammalian gene also encodes two isoforms, the larger of which is targeted to the nucleus, the other is found in both the nucleus and the cytoplasm. Also, like Dstpk61, $D p 70^{S 6 K}$ produces sex-enriched transcripts: the $3.7 \mathrm{~kb}$ transcript is most abundant in males, whereas the $2.8 \mathrm{~kb}$ transcript is most abundant in females (Stewart et al. 1996). The $5.0 \mathrm{~kb}$ transcript is expressed at similar levels in both sexes. Thus, it is possible that Dstpk61 and Dp70 $66 \mathrm{~K}$ are involved in a pathway that regulates sex-specific events.

SGK has also been shown to be a substrate of PDK1 (Kobayashi \& Cohen 1999, Park et al. 1999). The function of SGK is not clear, but it has been observed to undergo cytoplasmic-nuclear shuttling in synchrony with the cell cycle. This shuttling is differentially regulated depending on the proliferative state of the cell, suggesting that SGK may regulate cell proliferation (Park et al. 1999). No SGK homologue has been reported in Drosophila, and database searches do not identify possible candidate genes. Thus, it is possible that functions carried out by mammalian SGK might be mediated by a different kinase in Drosophila.

It has recently been shown that PDK1 also interacts with sphingosine, enabling it to phosphorylate a different target-PAK1 (King et al. 2000). Thus, interactions between PDK1 and different lipid components of the cell membrane may activate alternative signalling cascades, thereby eliciting different cellular responses. Similarly, it has been shown that phosphorylation of $\mathrm{p} 70^{\mathrm{S} 6 \mathrm{~K}}$ by PDK1 is not dependent on the presence of phospholipids (Alessi et al. 1998). Thus, cytoplasmic PDK1 may have yet another set of substrates.

PDK1 homologues have been identified in a number of organisms. S. cerevisiae has two PDK1 genes, PKH1 and $P K H 2$, which are required for cell growth and viability (Casamayor et al. 1999). Similarly, the S. pombe homo$\operatorname{logue}, \operatorname{ksg} 1$, is required for growth, and also for mating and sporulation (Niederberger \& Schweingruber 1999). The C. elegans homologue has multiple phenotypic effects, including the induction of diapause at the dauer larval stage and increased lifespan (Paradis et al. 1999). 
Biochemical analysis has identified a homologue in A. thaliana (AtPDK1) (Deak et al. 1999) and four PDK1 isoforms in sheep brains (Stephens et al. 1998). Perhaps the most extensively studied homologue is the mouse gene, $m P D K 1$. mPDK1 mRNAs appear to be expressed in a wide range of tissues, and produce multiple proteins ranging in size from 60 to $180 \mathrm{kDa}$. It also appears to encode a testis-specific transcript (Dong et al. 1999).

Dstpk61 is thought to be the Drosophila homologue of PDK1 (Alessi et al. 1997a). We have previously reported the presence of at least four different Dstpk61 transcripts: a common transcript; a female-specific carcass transcript; an ovary-specific transcript; and a testis-specific transcript (MacDougall et al. 1999). We also reported the sequence of three different cDNAs, one of which (EST40) is thought to represent the female-specific carcass transcript (MacDougall et al. 1999). This paper looks in detail at the different variants of RNA and protein generated by this gene in Drosophila and identifies some very intriguing results. This information is used to speculate on how the gene might function in different signalling pathways by producing transcripts and/or proteins that are localised to different regions of the cell. We also report the predicted structure of a mammalian PDK1 gene, and suggest that it too might produce multiple transcripts.

Drosophila is a very flexible model system, allowing information to be gathered by a variety of scientific approaches. Thus, a detailed analysis of Dstpk61 in Drosophila should aid the design of informative experiments to further elucidate the role of PDK1 in mammals. The publication of the Drosophila and human genomes and the development of expressed sequence tag (EST) databases in both systems should enable analysis of gene structure and the transcripts and proteins generated from a gene in Drosophila, a species amenable to genetic analysis, to help direct the search for protein isoforms with subtly different functions in humans. It is crucial that any isoforms are fully investigated, as they will provide the possibility of targeting a specific protein isoform for disease treatment. This may avoid the side effects which are bound to occur when a single gene participates in multiple signalling control pathways under a variety of cellular situations.

\section{Materials and Methods}

\section{Midi-preparation of plasmid DNA}

Hybaid (Ashford, Middlesex, UK) midi-prep kits were used to prepare up to $200 \mu \mathrm{g}$ plasmid DNA, following the manufacturer's instructions.

\section{Automated DNA sequencing}

Approximately $400 \mathrm{ng}$ high quality plasmid DNA were used as a template in sequencing reactions. $3 \cdot 2$ picomoles of the appropriate primer and $8 \mu \mathrm{l}$ reaction mix (ABI PRISM dye terminator cycle sequencing kit, PerkinElmer Corporation, Warrington, Cheshire, UK) and double distilled $\mathrm{H}_{2} \mathrm{O}$ were added to a final volume of $20 \mu \mathrm{l}$. The mixture was subjected to thermal cycling (Touchdown thermal cycler, Hybaid), as recommended by the Perkin-Elmer instruction manual. The program used is as follows: 25 cycles of $30 \mathrm{~s}$ denaturation at $96{ }^{\circ} \mathrm{C}$; $15 \mathrm{~s}$ at the appropriate primer annealing temperature; and a 4 min extension period at $60{ }^{\circ} \mathrm{C}$. The products were analysed using an ABI PRISM 377 DNA sequencer (Perkin-Elmer Corporation).

\section{Sequence analysis}

DNA sequences were analysed using GenejockeyII (Biosoft, Cambridge, UK) and the Genetics Computer Group (GCG; http://www.hgmp.msc.ac.uk) program package, version 10. BLAST searches (Altschul et al. 1990) were carried out using the NCBI server. This approach identified numerous EST clones and one genomic clone, BACR 48E09/AC006169. The structure of the gene was then deduced by comparing the cDNA sequences to the genomic sequence. Protein sequences were analysed for motifs using ProDom (Corpet et al. 1999, 2000), and subcellular localisation was predicted using PSORT (Nakai \& Kanehisa 1992).

\section{Results}

\section{Dstpk61 is represented 21 times in the EST database}

BLAST searches of the EST database were carried out using the sequence of $\mathrm{CDNA}^{\mathrm{B}}$ as a probe. This approach identified 21 ESTs. Sixteen EST cDNAs were identified from an embryonic library, four from the adult head library, and one from the adult ovarian library. Thus, Dstpk61 is expressed at different stages, and in multiple tissues, during development. We classified the ESTs into three distinct groups based on their $5^{\prime}$ sequence, as shown in Table 1.

The first exon of Group 1 ESTs, which we have named exon $1 \mathrm{a}$, is similar to that of $\mathrm{CDNA}^{\mathrm{B}}$ (see Fig. 1A $i$ ). This group contains LD16509 (EST09), which we have previously sequenced and published (MacDougall et al. 1999). EST09 is thought to be a more complete version of cDNA $^{\mathrm{B}}$. We have sequenced a further two Group 1 cDNAs, LD19385 (EST85) and LD32017 (EST17). These cDNAs appear to represent alternative splice forms of Dstpk61.

Group 2 ESTs contain a novel first exon, which we have termed exon $1 \mathrm{~b}$ (see Fig. 1Aii). Exon $1 \mathrm{~b}$ is located approximately $3 \mathrm{~kb}$ upstream of exon $1 \mathrm{a}$. We have previously reported the sequence of LD19940 (EST40), which we believe to be a female-specific carcass transcript 
Table 1 CDNA libraries from which EST sequences have been derived

\begin{tabular}{|c|c|}
\hline Name & Source \\
\hline $\mathrm{HL}$ & Adult heads \\
\hline $\mathrm{GH}$ & Adult heads \\
\hline GM & Ovaries, stage $1-6$ \\
\hline LP & Various larval stages and early pupae \\
\hline LD & Embryos, $0-22 \mathrm{~h}$ \\
\hline $\mathrm{CK}$ & $\begin{array}{l}\text { Rough endoplasmic reticulum } \\
\text { from } 8-16 \text { h embryos }\end{array}$ \\
\hline SD & Drosophila tissue culture cells \\
\hline
\end{tabular}

\section{Cloning information}

$\overline{\text { EcoR I/Xho I sites of Uni-Zap XR or pOT2 }}$

EcoR I/Xho I sites of Uni-Zap XR or pOT2

EcoR I/Xho I sites of Uni-Zap XR or pOT2 EcoR I/Xho I sites of pOT2 EcoR I/Xho I sites of Uni-Zap XR or pOT2 Hind III/Pst I sites of pBluescript SK(+)

EcoR I/Xho I sites of pOT2

\section{Comments}

Many clones not full-length or have no inserts

Separate from HL library. Made because many HL clones were not full-length or had no inserts

Biased for mRNAs encoding secreted and membrane-bound proteins
(MacDougall et al. 1999). A further two Group 2 cDNAs have now been sequenced, LD02269 (EST69) and GM04058 (EST58). EST69 is thought to represent the alternatively spliced non-sex-specific carcass transcript reported in MacDougall et al. (1999).

Group 3 ESTs contain neither exon 1a nor exon $1 \mathrm{~b}$ (Fig. 1Aiii). These cDNAs contain either a novel first exon (GH15751, LD38659, and LD13752), or start transcription midway through a previously characterised exon (LD13190 and LD46834). In the latter case, it is possible that these cDNAs are truncated at their $5^{\prime}$ ends.

\section{Dstpk61 expression is regulated in many different ways}

Based on the above sequence data, we have determined that the Dstpk61 locus consists of at least 11 different exons and produces at least 10 different transcripts (see Fig. 1B). This variety is achieved by using alternative promoters, alternative polyadenylation sites, and alternative splice sites.

Group 1 transcripts are likely to be produced from one promoter, which we previously termed $\mathrm{P}^{09}$ (MacDougall et al. 1999). Likewise, it is probable that Group 2 transcripts all use a second distinct promoter, termed $\mathrm{P}^{40}$ in our earlier publication (MacDougall et al. 1999). Group 3 consists of five ESTs, all of which appear to be transcribed from different promoters. However, it is possible that LD46834 may represent a $5^{\prime}$ truncated version of LD13190, which may itself be a $5^{\prime}$ truncation of any of the other cDNAs. Similarly, it is not clear if $\mathrm{cDNA}^{\mathrm{A}}$ is truncated and uses $\mathrm{P}^{09}$, or if it represents a full-length transcript transcribed from a different promoter. Thus, at least five, and possibly eight, different promoters are used to produce mRNAs from the Dstpk61 locus (see Fig. 1B).

Different polyadenylation sites are also used in different transcripts (see Fig. 1B). At least 10 potential polyadenylation signals have been delimited in the $3^{\prime}$ sequence of Dstpk61 (MacDougall et al. 1999). Six different polyadenylation signals are used in the eight cDNAs that have been fully sequenced, and we have recently isolated an additional two cDNAs that use two further poly(A) sites (Sathirana, T Wood, D Clyde \& M Bownes, unpublished observations). It is likely that all 10 potential poly(A) signals will be used in vivo to produce transcripts that have variable $3^{\prime}$ untranslated regions (UTRs).

Alternative splicing is also used to further increase the number of transcripts from the Dstpk61 locus. Exon 1a contains three different splice donor sites, giving rise to three alternative forms of this exon. Also, EST40 and GM04058 are spliced differently to LD02269, which does not contain exons 2 and 3 . This splice event is likely to be regulated in a sex-specific manner in adult carcass tissue EST40 (or a similar Group 2 transcript) is detected in female, but not male, carcass RNA by RT-PCR, whereas EST69 is detected in carcass RNA from both sexes (MacDougall et al. 1999).

Considering the variety of promoters, splice sites, and polyadenylation sites available, it is possible that the transcripts we have described represent only a subset of those produced from Dstpk61 in the cell.

\section{Dstpk61 transcripts encode at least four different protein isoforms}

Despite the large number of potential Dstpk61 transcripts described above, they encode only four predicted protein isoforms (see Fig. 2). EST40 and EST58 encode an 836 amino acid protein, DSTPK61 $1^{836}$, the largest of the four isoforms. GH15751 encodes a slightly smaller protein of 819 amino acids (DSTPK61 ${ }^{819}$ ), which lacks the 22 most N-terminal amino acids of DSTPK61 $1^{836}$ and replaces them with the five amino acid sequence MNIIQ. The majority of the predicted transcripts $\left(\mathrm{cDNA}^{\mathrm{B}} / \mathrm{EST}\right.$ 9, cDNA $^{A}$, EST17, EST85, LD13190, and LD46834) encode a 755 amino acid protein, DSTPK61 $1^{755}$. DSTPK61 $61^{755}$ is identical to DSTPK61 ${ }^{836}$ except that it lacks 81 amino acids from the $\mathrm{N}$-terminus. The remaining transcripts (EST69, LD38659, and LD13752) encode a 539 amino acid protein, DSTPK61 ${ }^{539}$, which is identical to the most C-terminal 539 amino acids of the other three isoforms. Raising antibodies to bacterially produced 
(A) $i \quad$ Group 1 EST sequence in database

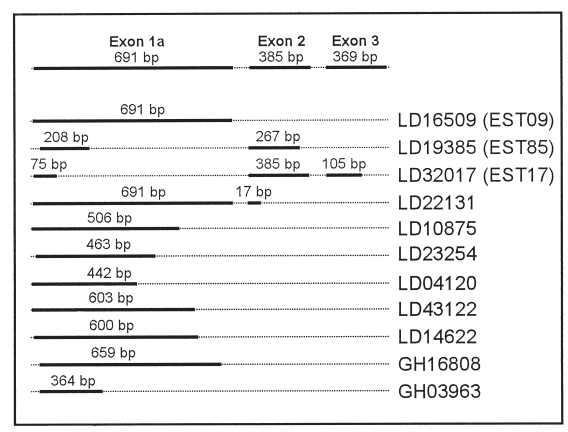

ii Group 2 EST sequence in database

\begin{tabular}{|c|c|c|c|}
\hline $\begin{array}{c}\text { Exon 1b } \\
415 \mathrm{bp} \\
\end{array}$ & $\begin{array}{r}\text { Exon } 2 \\
385 \text { bp }\end{array}$ & $\begin{array}{r}\text { Exon } 3 \\
369 \mathrm{bp} \\
\end{array}$ & \multirow{6}{*}{$\begin{array}{l}\text { GM04058 (EST58) } \\
\text { LD19940 (EST40) } \\
\text { LD02269 (EST69) } \\
\text { LD43995 } \\
\text { GH02335 }\end{array}$} \\
\hline $415 \mathrm{bp}$ & $159 \mathrm{bp}$ & & \\
\hline $405 \mathrm{bp}$ & $31 \mathrm{bp}$ & & \\
\hline $406 \mathrm{bp}$ & \multicolumn{2}{|c|}{$60 \mathrm{bp}$} & \\
\hline $413 \mathrm{bp}$ & $188 \mathrm{bp}$ & & \\
\hline $322 \mathrm{bp}$ & $309 \mathrm{bp}$ & & \\
\hline
\end{tabular}

iii Group 3 EST sequence in database

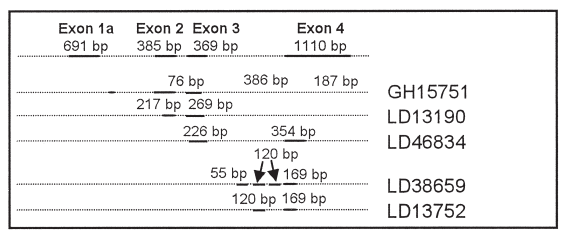

(B)
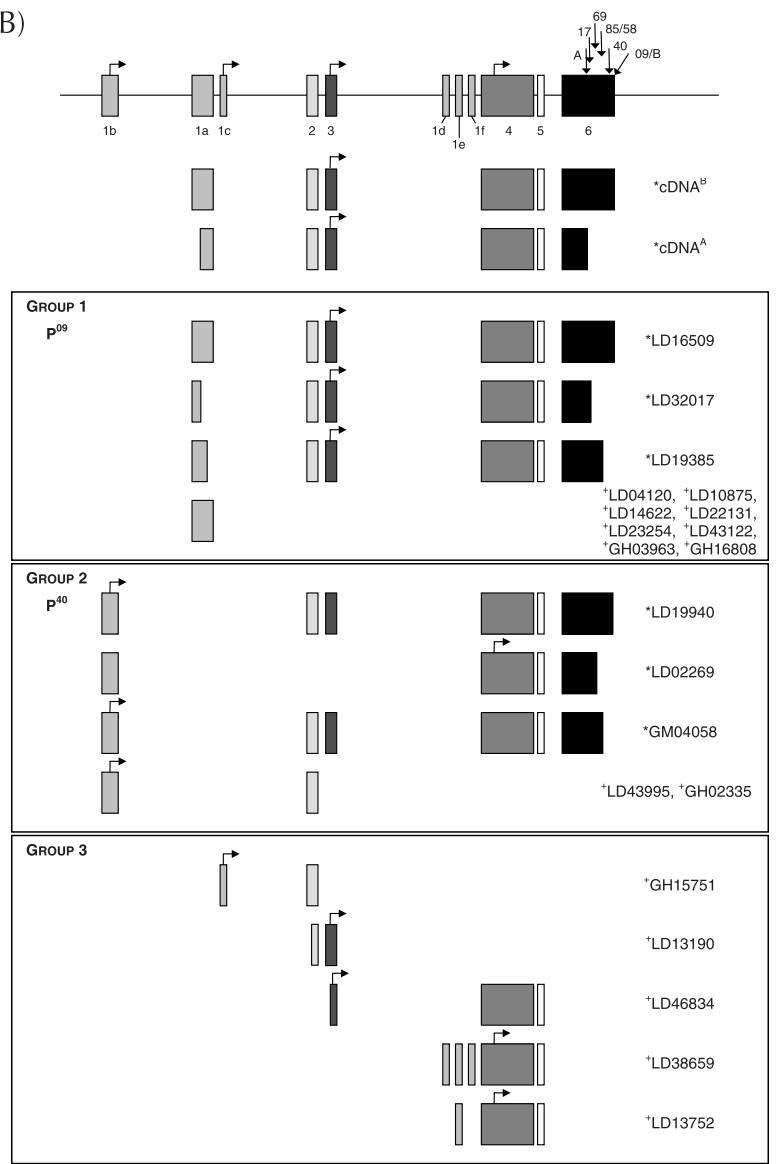

Figure 1 (A) Diagram showing the 5' structures of EST sequences with homology to Dstpk61. These ESTs have been subdivided into three groups. (i) Group 1 EST cDNA sequence, compared with exons 1a, 2 and 3 of Dstpk61. (ii) Group 2 EST cDNA sequence, compared with exons $1 \mathrm{~b}, 2$ and 3 of Dstpk61. (iii) Group 3 EST cDNA sequence, compared with exons 1a, 2, 3 and 4 of Dstpk61. (B) Diagram showing the exon structure of the Dstpk61 locus and EST cDNAs. cDNAs marked * have been fully sequenced, the sequence of those marked + has been taken from the EST database. Potential translational start sites are marked with a bent arrow $(\vec{\Gamma})$, and polyadenylation sites are marked with straight arrows $(\downarrow)$. $\mathrm{LD}=$ embryonic library, $\mathrm{GH}=$ adult head library, $\mathrm{GM}=$ ovary library.

recombinant DSTPK61 ${ }^{755}$ (Alessi et al. 1997a) and to peptides from various regions of the proteins will enable us to determine the distribution of these isoforms in Drosophila tissues.

\section{DSTPK61 contains multiple protein domains}

We have previously shown that DSTPK61 ${ }^{755}$ contains three distinct domains: an OPA repeat (or CAX) domain; a Pleckstrin Homology (PH) domain; and a kinase domain (Alessi et al. 1997a, MacDougall et al. 1999). We have also shown that recombinant DSTPK61 $1^{755}$ is capable of phosphorylating $\mathrm{PKB}$ in vitro in a manner similar to its proposed human homologue, PDK1 (Alessi et al. 1997a). A ProDom (Corpet et al. 1999, 2000) search was carried out to identify further motifs and domains. The amino acid sequence of each isoform was also analysed using the PSORT program (Nakai \& Kanehisa 1992) to determine its most likely subcellular location. It was hoped these approaches might help elucidate the functional significance of the alternative protein isoforms.

The ProDom results revealed the presence of a putative DNA binding domain immediately $\mathrm{N}$-terminal of the OPA repeat domain and a bipartite nuclear localisation signal (see Fig. 2). PSORT confirmed that all four isoforms are likely to be nuclear (see Fig. 2). However, DSTPK $61^{836}$ also contains a putative $\mathrm{N}$-terminal signal found in proteins that are located in the mitochondrial intermembrane space (see Fig. 2). This sequence is not present in the other three isoforms. The shortest of the four proteins, DSTPK61 ${ }^{539}$, lacks part of the kinase domain and one part of the bipartite nuclear localisation signal. 


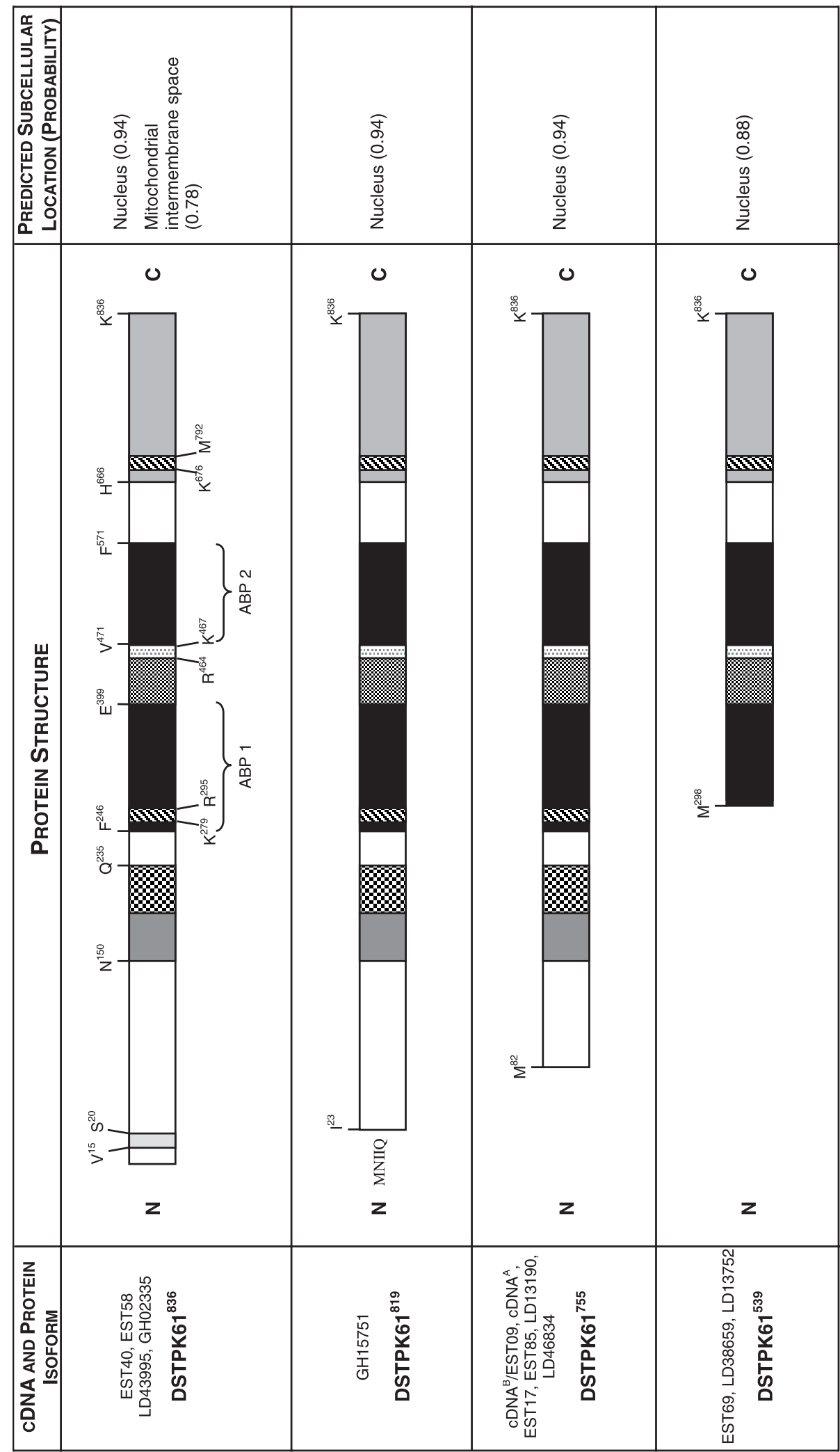

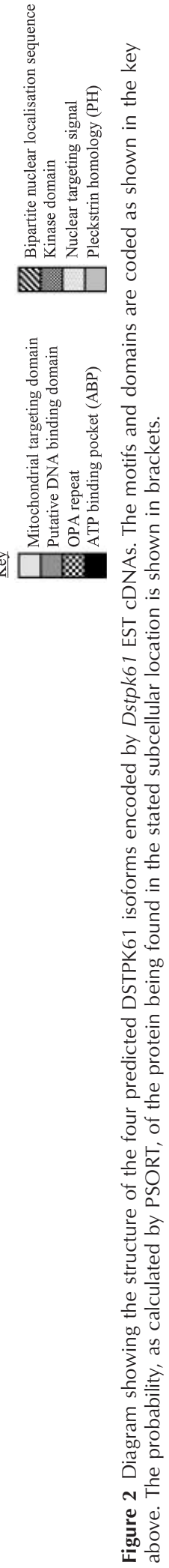




\section{PDK1 is likely to produce multiple transcripts}

Three identical 1891 nucleotide human PDK1 mRNA sequences (Accession numbers Y15056, AF017995, and NM_002613), which encode a 555 amino acid protein, have been submitted to the sequence database. These mRNAs show homology to the sequence contained in the P1 clone, AC005591, which maps to human chromosome 16. This contradicts genetic mapping information, which places PDK1 on human chromosome 22.

Comparison of the mRNA sequence in the database to the genomic sequence reveals that the gene is composed of 14 exons, the first of which is not contained within the P1 clone. However, it appears that the clone contains two copies of exons $7-10$, and exons $2-10$ are in the opposite orientation to exons 7-14 (see Fig. 3A). It is possible that the P1 clone contains a PDK1 pseudogene resulting from a partial gene duplication event during evolution, but the rearrangement could also be an artefact of the cloning and sequencing processes.

The genomic sequence was scanned for additional potential exons using the FGENESH program (Smith et al. 1996), which identified 23 putative exons not contained within the published mRNA sequence (see Fig. 3A). Six of these exons are likely to represent a distinct gene downstream of PDK1, which is transcribed in the opposite direction (see Fig. 3B). It is possible that the other exons could be included in PDK1 mRNAs by alternative splicing to produce alternative PDK1 transcripts. However, it should be noted that gene prediction programs have their limitations. Indeed, FGENESH failed to identify some of the exons used in the published PDK1 mRNA sequence.

It is very likely that a number of $P D K 1$ transcripts are present within the cell. At least 116 EST cDNAs have been classified as potential PDK1 transcripts. These cDNAs range in size from 0.3 to $4.2 \mathrm{~kb}$, and have been isolated from many different tissues, including eye, testes, ovary, brain and blood. Those that have been sequenced appear to share identical $3^{\prime}$ ends, although the $5^{\prime}$ ends appear to differ. Many of the smallest clones are likely to be truncated. However, some of the smaller species will not be truncated, as the $5^{\prime}$ sequence of the larger cDNAs is often contained within the smaller cDNAs. This observation suggests that the larger cDNAs may be produced from alternative internal promoters and may contain additional exons. Thus, it is very likely that, as for Dstpk61, multiple PDK1 protein isoforms exist, as the additional exons are likely to be introduced into protein-coding regions.

\section{Discussion}

What is the biological significance of alternative Dstpk61 transcripts?

Although PDK1 homologues exist in a number of organisms, the gene structure and transcriptional activity of these loci have not been studied in detail. To date, only $m P D K 1$ has been shown to produce multiple transcripts and protein isoforms (Dong et al. 1999). No alternative functions have been attributed to the variant mRNAs or proteins, nor has their cellular distribution been reported.

Dstpk61 is the Drosophila homologue of PDK1, and we have shown that this locus produces multiple transcripts. Some of these encode different protein isoforms, the significance of which will be discussed in the next section. However, some transcripts encode identical proteins: the mRNAs differ only in the nucleic acid sequence of their $5^{\prime}$ and/or 3' UTRs. Why do these variant transcripts exist?

Differences in 5' UTRs occur due to the use of different promoters. These promoters may be regulated by different trans-acting factors. The activity of a given promoter will depend on the availability of the appropriate regulatory proteins, some of which may be present only in certain tissues and/or at certain developmental stages. This may allow their expression to be co-ordinated with other genes in specific response pathways in different combinations in different cells, or at different times.

Similarly, alternative splicing of Dstpk61 transcripts could be regulated by splicing factors with restricted expression patterns. Thus, some Dstpk61 transcripts may be expressed in a temporally and/or spatially restricted manner. Indeed, we have previously reported the presence of a female carcass-specific Dstpk61 transcript that presumably arises from alternative splicing. Many examples exist in Drosophila of splicing factors with restricted expression domains. For example, somatic sex is determined in flies by a series of alternative splicing events that are regulated by the female-specific splicing factors, SXL and TRA (Boggs et al. 1987, Hoshijima et al. 1991). Splicing of the homeotic gene, Ultrabithorax $(U b x)$, is also regulated in a tissue and temporal-specific manner (O'Connor et al. 1988).

The $5^{\prime}$ and $3^{\prime}$ UTRs may also contain binding sites for trans-acting factors (proteins or RNAs) that regulate translation or the subcellular localisation of the transcript. Again, this might occur in a spatially or temporally dependent manner according to the distribution of the relevant trans-acting factors. For example, many spermspecific transcripts in Drosophila contain Translational Control Elements (TCEs) in their 5' UTRs, which ensure that translation is repressed until the appropriate stage of spermatogenesis (for review see Schäfer et al. 1995). Drosophila development also requires certain transcripts to be specifically localised. A well understood example is the localisation of bicoid and oskar mRNAs during embryogenesis and prospero during nervous system development (Campos-Ortega 1997). STAUFEN is crucial for this localisation by binding to the 3' UTRs of the mRNAs (for review on RNA localisation see Bashirullah et al. 1998). 


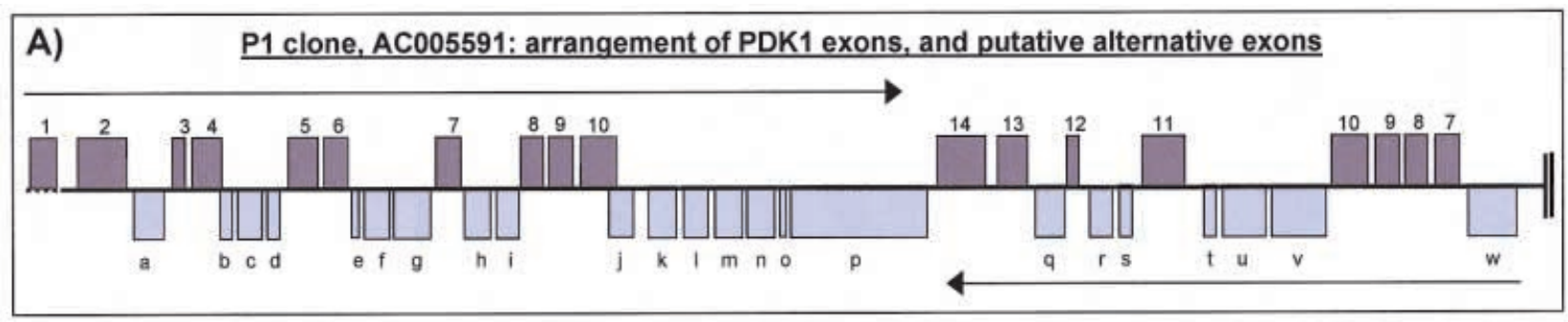

\section{B)}

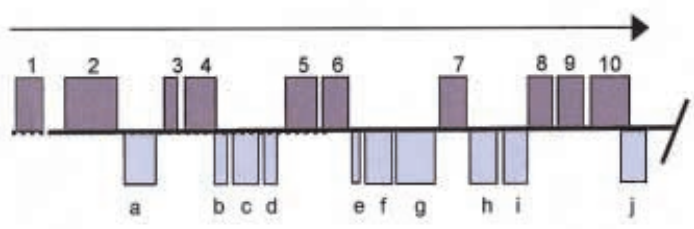

Putative corrected structure of P1 clone, AC005591, and the PDK1 gene

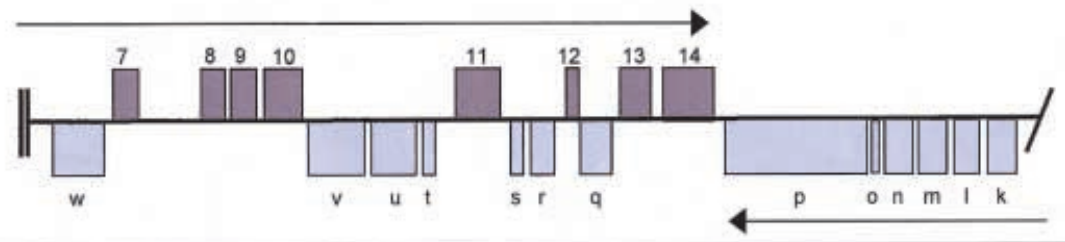

Figure 3 Diagram showing the putative structure of the human PDK1 locus on P1 clone AC005591, which has been mapped to chromosome 16. Exons found in published mRNA sequences are numbered 1-14. Alternative exons, as predicted by FGENESH, are denoted $\mathrm{a}-\mathrm{w}$. Arrows denote direction in which exons are transcribed. (A) Exon arrangement of human PDK1 according to the AC005591 sequence that appears in the database. (B) Putative corrected arrangement of the human PDK1 locus, assuming that AC005591 has undergone genomic rearrangements during the cloning and sequencing processes.

What is the biological significance of alternative DSTPK61 protein isoforms?

Proteins are modular structures that consist of distinct domains with discrete functions. Specific types of domains can be combined to form proteins with very specialised capabilities. Alternative splicing is one method whereby different protein domains can be included or excluded from a family of proteins encoded by a single locus, thereby conferring on them different cellular functions. This is one possible explanation for the production of multiple Dstpk61 transcripts, which encode four protein isoforms.

The four DSTPK61 isoforms contain all or some of the following domains and motifs: a kinase domain; a Pleckstrin Homology (PH) domain; an OPA repeat/DNA binding domain; nuclear localisation signals; and a mitochondrial intermembrane space targeting sequence.

The kinase domain is essential for the role of DSTPK61 as a signalling molecule. It contains two putative ATP binding pockets, which are responsible for the phosphorylation of substrate molecules. DSTPK61 $1^{836}$, DSTPK61 ${ }^{819}$ and DSTPK61 $61^{755}$ have identical kinase domains. DSTPK61 ${ }^{539}$ lacks $52 \mathrm{~N}$-terminal amino acids of the kinase domain, disrupting one of the two ATP binding pockets. It is not clear if this isoform will be kinase dead, or will have an altered substrate specificity. In the long term, this could be investigated by producing this form of the kinase in vitro and checking its biochemical activity.

The $\mathrm{PH}$ domain is involved in membrane and protein:protein interactions. In mammals, the $\mathrm{PH}$ domain of PDK1 interacts with phosphoinositol lipids $\left(\mathrm{PI}(3,4) \mathrm{P}_{2}\right.$ and $\left.\operatorname{PI}(3,4,5) \mathrm{P}_{3}\right)$ at the cell membrane, increasing its ability to activate certain substrates, such as PKB (Alessi et al. 1997a). Recombinant DSTPK61 ${ }^{755}$ has also been shown to activate $\mathrm{PKB}$ in a $\mathrm{PI}(3,4) \mathrm{P}_{2}$ and $\mathrm{PI}(3,4,5) \mathrm{P}_{3}$ dependent manner (Alessi et al. 1997a). However, PDK1 can also interact with other lipids, such as sphingosine, allowing it to phosphorylate different substrates (King et al. 2000). These interactions presumably occur via the PH domain. All four DSTPK61 isoforms contain identical PH domains. It is possible, nevertheless, that different isoforms interact with different lipids or proteins dependent on their tissue distribution and subcellular localisation.

The OPA repeat (or CAX) domain is a stretch of glutamine residues found in an array of proteins in many 
organisms, from yeast to humans. In Drosophila, OPA repeats are found mainly in proteins with developmentally restricted expression patterns, for example, the neurogenic protein Notch. Many OPA repeat containing proteins also have DNA binding domains, for example the homeobox proteins in Drosophila, which are transcriptional regulators (Biggin \& McGinnis 1997). The three largest DSTPK61 isoforms (DSTPK61 ${ }^{836}$, DSTPK61 ${ }^{819}$ and DSTPK61 ${ }^{755}$ ) all contain an OPA repeat and upstream putative DNA binding domain. However, DSTPK61 ${ }^{539}$ lacks these domains, suggesting that it might have a ubiquitous expression domain with a more general cellular role, possibly functioning in the cytoplasm. This is in keeping with RT-PCR analysis, which suggests that the transcript represented by EST69 is expressed in both male and female carcasses (MacDougall et al. 1999).

PSORT analysis of the four DSTPK61 isoforms suggests that they all have a high probability of being localised to the nucleus. This theory is substantiated by the presence of two types of nuclear localisation signals. One targeting sequence is located within the kinase domain. The other is a bipartite signal, one part of which is situated at the $\mathrm{N}$-terminus of the kinase domain, with the other part at the C-terminal end of the kinase domain. The three larger DSTPK61 isoforms contain all these targeting sequences, and have a probability of 0.94 of being nuclear. What might be the functional relevance of nuclear DSTPK61 localisation?

It is possible that DSTPK61 has different substrates and/or cellular responses in the nucleus. Indeed, it may be that different DSTPK61 isoforms have slightly different tertiary structures. Thus, some isoforms might be targeted directly to the nucleus. However, the nuclear targeting signals might be hidden in other isoforms and they might translocate to the nucleus only when they interact with specific substrates.

DSTPK61 ${ }^{539}$ lacks the N-terminal part of the bipartite nuclear localisation signal. The predicted probability of this isoform being located in the nucleus is $0 \cdot 88$. Again, this observation suggests that DSTPK61 ${ }^{539}$ might be less restricted in its function and location than the other isoforms.

PSORT analysis also identified an N-terminal motif in DSTPK $61^{836}$ that targets proteins to the mitochondrial intermembrane space. The other DSTPK61 isoforms lack this sequence. It is interesting that DSTPK61 ${ }^{836}$ is encoded by transcripts that have been detected in female carcass tissue and ovaries, but not in male carcasses. This might reflect the higher energy requirement of females. However, it is interesting to note that the potential DSTPK61 substrate, PKC, is located in the mitochondria, where it has a role in promoting cell survival (for review, see Cross et al. 2000). It could be that it is via this substrate in mitochondria that DSTPK61 can affect cell survival. There are differences in the nerve cell divisions in males and females. Also, interactions are needed between somatic cells and germ cells of the female for germline sex determination to occur and for oogenesis to be initiated (Steinmann-Zwicky et al. 1989, Horabin et al. 1995).

Experiments with Drosophila should reveal the functions of DSTPK61 and PDK1

As yet, little is known about Dstpk61 function in Drosophila, and much work remains to be done. Recombinant DSTPK $61^{755}$ has already been shown to possess kinase activity (Alessi et al. 1997a). Kinase assays should be carried out on the other three isoforms. It will be particularly interesting to determine if DSTPK61 ${ }^{539}$, which lacks part of the kinase domain, retains kinase activity.

Raising antibodies to different regions of the DSTPK61 isoforms should allow their tissue and developmental expression profiles to be determined, and will confirm which isoforms are found in vivo. Antibodies could also be used for immuno-co-precipitation experiments to identify potential DSTPK61 substrates.

The subcellular localisation of the various protein isoforms should also be investigated - are all isoforms found in the nucleus, and is DSTPK61 ${ }^{836}$ found in the mitochondrial intermembrane space? The in vivo localisation of the various isoforms can be studied by making GFP-tagged recombinant proteins. It will also be interesting to determine if DSTPK61 ${ }^{836}$ can be detected in purified mitochondria.

Currently, we are undertaking screens for mutations in the Dstpk61 locus, to enable us to investigate their phenotypes. Dstpk61 is expressed in early embryos, so it is possible that Dstpk61 null individuals will die early in development. However, it may be possible to create viable fly lines that lack specific transcripts or protein isoforms, which should help determine if different isoforms or transcripts are responsible for different aspects of Dstpk61 function. Transgenic technology will also allow us to over-express, or 'knock-out', Dstpk61 function at later developmental stages if viable mutant lines cannot be raised.

Genetic studies should clarify the in vivo roles of DSTPK61 and help identify its substrates and the pathways in which it is involved. This information should aid the design of more informed experiments in mammalian systems that do not have the experimental flexibility of Drosophila.

\section{Acknowledgements}

The authors wish to thank Sheila Milne for help in preparing this manuscript, Kevin Leaper for helpful comments and advice, Nicola Preston for assistance with 
automated sequencing, and Steven Addicott for assistance with diagrams.

\section{References}

Alessi DR, Deak M, Casamayor A, Caudwell FB, Morrice N, Norman DG, Gaffney P, Reese CB, MacDougall CN, Harbison D, Ashworth A \& Bownes M 1997a 3-Phosphoinositide-dependent protein kinase-1 (PDK1): structural and functional homology with the Drosophila DSTPK61 kinase. Current Biology 7 776-789.

Alessi DR, James SR, Downes CP, Holmes AB, Gaffney PR, Reese CB \& Cohen P 1997b Characterisation of a 3-phosphoinositidedependent protein kinase which phosphorylates and activates protein kinase B alpha. Current Biology 7 261-269.

Alessi DR, Kozlowski MT, Weng QP, Morrice N \& Avruch J 1998 3-Phosphoinositide-dependent protein kinase 1 (PDK1) phosphorylates and activates the p70 S6 kinase in vivo and in vitro. Current Biology 8 69-81.

Altschul SF, Gish W, Miller W, Myers EW \& Lipman DJ 1990 Basic local alignment search tool. Journal of Molecular Biology 215 403-410.

Bashirullah A, Cooperstock RL \& Lipshitz HD 1998 RNA localization in development. Annual Review of Biochemistry 67 335-394.

Biggin MD \& McGinnis W 1997 Regulation of segmentation and segmental identity by Drosophila homeoproteins: the role of DNA binding in functional activity and specificity. Development 124 4425-4433.

Boggs RT, Gregor P, Idriss S, Belote JM \& McKeown M 1987 Regulation of sexual differentiation in D. melanogaster via alternative splicing of RNA from the transformer gene. Cell 50 739-747.

Bohni R, Riesgo-Escovar J, Oldham S, Broglio W, Stocker H, Andruss BF, Beckingham K \& Hafen E 1999 Autonomous control of cell and organ size by CHICO, a Drosophila homolog of vertebrate IRS1-4. Cell 97 865-875.

Brunet A, Bonni A, Zigmond MJ, Lin MZ, Juo P, Hu LS, Anderson MJ, Arden KC, Blenis J \& Greenberg ME 1999 Akt promotes cell survival by phosphorylating and inhibiting a Forkhead transcription factor. Cell 96 857-868.

Campos-Ortega JA 1997 Asymmetric division: dynastic intricacies of neuroblast division. Current Biology 7 R726-728.

Casamayor A, Torrance PD, Kobayashi T, Thorner J \& Alessi DR 1999 Functional couterparts of mammalian protein kinases PDK1 and SGK in budding yeast. Current Biology 9 186-197.

Cheng X, Ma Y, Moore M, Hemmings BA \& Taylor SS 1998 Phosphorylation and activation of cAMP-dependent kinase by phosphoinositide-dependent protein kinase. PNAS 95 9849-9854.

Corpet F, Gouzy J \& Kahn D 1999 Recent improvements of the ProDom database of protein domain families. Nucleic Acids Research 27 263-267.

Corpet F, Servan F, Gouzy J \& Kahn D 2000 ProDom and ProDom-CG: tools for protein domain analysis and whole genome comparisons. Nucleic Acids Research 28 267-269.

Craddock BL, Orchiston EA, Hinton HJ \& Welham MJ 1999 Dissociation of apoptosis from proliferation, protein kinase B activation, and BAD phosphorylation in interleukin-3-mediated phosphoinositide 3-kinase signalling. Journal of Biological Chemistry 274 10633-10640.

Cross TG, Scheel-Toellner D, Henriquez NV, Deacon E, Salmon M \& Lord JM 2000 Serine/Threonine protein kinases and apoptosis. Experimental Cell Research 256 34-41.

Currie RA, Walker KS, Gray A, Deak M, Casamayor A, Downes CP, Cohen P, Alessi DR \& Lucocq J 1999 Role of phosphatidylinositol 3,4,5-triphosphate in regulating the activity and localisation of 3-phosphoinositide-dependent protein kinase-1. Biochemical Journal 337 575-585.

Deak M, Casamayor A, Currie RA, Downes CP \& Alessi DR 1999 Characterisation of a plant 3-phosphoinositide-dependent protein kinase-1 homologue which contains a pleckstrin homology domain. FEBS Letters 451 220-226.

Dong LQ, Zhang RB, Langlais P, He H, Clark M, Zhu L \& Liu F 1999 Primary structure, tissue distribution, and expression of mouse phosphoinositide-dependent protein kinase-1, a protein kinase that phosphorylates and activates protein kinase Czeta. Journal of Biological Chemistry 274 8117-8122.

Horabin JI, Bopp D, Waterbury J \& Schedl P 1995 Selection and maintenance of sexual identity in the Drosophila germline. Genetics 141 1521-1535.

Hoshijima K, Inoue K, Higuchi I, Skakmoto H \& Shimura Y 1991 Control of doublesex alternative splicing by transformer and transformer-2 in Drosophila. Science 252 833-836.

Kalderon D 1997 Hedgehog signalling: Ci complex cuts and clasps. Current Biology 7 R759-762.

King CC, Zenke FT, Dawson PE, Dutil EM, Newton AC, Hemmings BA \& Bokoch GM 2000 Sphingosine is a novel activator of 3-phosphoinositide-dependent kinase-1. Journal of Biological Chemistry 275 18108-18113.

Kobayashi T \& Cohen P 1999 Activation of serum- and glucocorticoidregulated protein kinase by agonists that activate phosphatidylinositide3-kinase is mediated by 3-phosphoinositide-dependent protein kinase-1 (PDK1) and PDK2. Biochemical Journal 339 319-328.

Leevers SJ, Weinkove D, MacDougall LK, Hafen E \& Waterfield MD 1996 The Drosophila phosphoinositide 3-kinase Dp110 promotes cell growth. EMBO Journal 15 6584-6594.

Le Good JA, Ziegler WH, Parekh DB, Alessi DR, Cohen P \& Parker PJ 1998 Protein kinase C isotypes controlled by phosphoinositide 3-kinase through the protein kinase PDK1. Science 281 2042-2045.

Liu WS \& Heckman CA 1998 The sevenfold way of PKC regulation. Cell Signal 10 529-542.

MacDougall CN, Clyde D, Wood T, Todman M, Harbison D \& Bownes M 1999 Sex-specific transcripts of the Dstpk61 serine/ threonine kinase in Drosophila melanogaster. European Journal of Biochemistry 262 456-466.

Nakai K \& Kanehisa M 1992 A knowledge base for predicting protein localization sites in eukaryotic cells. Genomics 14 897-911.

Niederberger C \& Schweingruber ME 1999 A Schizosaccharomyces pombe gene, $k s g l$, that shows structural homology to the human phosphoinositide-dependent protein kinase PDK1, is essential for growth, mating and sporulation. Molecular and General Genetics 261 177-183.

O'Connor MB, Binari R, Perkins LA \& Bender W 1988 Alternative RNA products from the Ultrabithorax domain of the bithorax complex. EMBO Journal 7 435-445.

Ohlmeyer JT \& Kalderon D 1997 Dual pathways for induction of wingless expression by protein kinase A and Hedgehog in Drosophila embryos. Genes and Development 11 2250-2258.

Paradis S, Ailion M, Tokes A, Thomas JH \& Ruvkun G 1999 A PDK1 homolog is necessary and sufficient to transduce AGE-1 P13 kinase signals that regulate diapause in Caenorhabditis elegans. Genes and Development 13 1438-1452.

Park J, Leong ML, Buse P, Maiyar AC, Firestone GL \& Hemmings BA 1999 Serum and glucocorticoid-inducible kinase (SGK) is a target of the PI 3-kinase-stimulated signalling pathway. EMBO Journal 18 3024-3033.

del Peso L, Gonzalez-Garcia M, Page C, Herrera R \& Nunez G 1997 Interleukin-3-induced phosphorylation of BAD through the protein kinase Akt. Science 278 687-689.

Peterson RT \& Schreiber SL 1998 Translation control: connecting mitogens and the ribosome. Current Biology 8 R248.

Pullen N, Dennis PB, Andjelkovich M, Dufner A, Kozma SC, Hemmings BA \& Thomas G 1998 Phosphorylation and activation of p70s6k by PDK1. Science 279 707-710.

Schäfer M, Nayernia K, Engel W \& Schäfer U 1995 Translational control in spermatogenesis. Developmental Biology 172 344-352. 
Scheid MP \& Duronio V 1998 Dissociation of cytokine-induced phosphorylation of Bad and activation of $\mathrm{PKB} / \mathrm{akt}$ : involvement of MEK upstream of Bad phosphorylation. PNAS 95 7439-7444.

Sibilia M \& Wagner EF 1995 Strain-dependent epithelial defects in mice lacking the EGF receptor. Science 269 234-238.

Skalhegg BS \& Tasken K 1997 Specificity in the cAMP/PKA signaling pathway. Differential expression, regulation, and subcellular localization of subunits of PKA. Frontiers of Bioscience 2 d331-342.

Smith RF, Weise BA, Wojzynski MK, Davison DB \& Worley KC 1996 BCM Search Launcher - an integrated interface to molecular biology data base search and analysis services available on the World Wide Web. Genome Research 6 454-462.

Staveley BE, Ruel L, Jin J, Stambolic V, Mastronardi FG, Heitzler P, Woodgett JR \& Manoukian AS 1998 Genetic analysis of protein kinase B (AKT) in Drosophila. Current Biology 8 599-602.

Steinmann-Zwicky M, Schmid H \& Nothiger R 1989 Cellautonomous and inductive signals can determine the sex of the germ line of Drosophila by regulating the gene Sxl. Cell 57 157-166.

Stephens L, Anderson K, Stokoe D, Erdjument-Bromage H, Painter GF, Holmes AB, Gaffney PRJ, Reese CB, McCormick F, Tempst P, Coadwell J \& Hawkins PT 1998 Protein kinase B kinases that mediate phosphatidylinositol 3,4,5-triphosphate-dependent activation of protein kinase B. Science 279 710-714.

Stewart MJ, Berry COA, Zilberman F, Thomas G \& Kozma SC 1996 The Drosophila $p 70(s 6 k)$ homolog exhibits conserved regulatory elements and rapamycin sensitivity. PNAS 93 10791-10796.

Van Buskirk C \& Schupbach T 1999 Versatility in signalling: multiple responses to EGF receptor activation during Drosophila oogenesis. Trends in Cell Biology 9 1-4.

Watson KL, Chou MM, Blenis J, Gelbart WM \& Erikson RL 1996 A Drosophila gene structurally and functionally homologous to the mammalian 70-kDa S6 kinase gene. PNAS 93 13694-13698.

Zhong H, SuYang H, Erdjument-Bromage H, Tempst P \& Ghosh S 1997 The transcriptional activity of NF-kappa B is regulated by the I kappa B-associated PKAc subunit through a cyclic AMPindependent mechanism. Cell 89 413-424.

Received 30 May 2000

Revised manuscript received 17 July 2000

Accepted 22 August 2000 